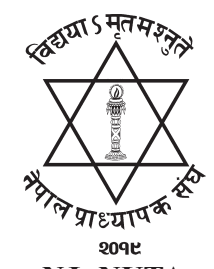

NJ: NUTA

\title{
Positional Number System
}

\author{
Anil Chandra Jha \\ Balmiki Campus, Nepal Sanskrit University Nepal \\ Email: jha.anilchandra3@gmail.com
}

\begin{abstract}
In a positional number system, the numerical value of each symbol depends on its position in the sequence of digits representing the number. Any integer greater than 1 may serve as a base, and in a base $b$ system there are $b$ digits represented conventionally by the digits $0,1,2, \ldots, b-1$. In this paper we introduce the following four positional number systems: decimal (base-10), binary (base-2), Octal (base-8) and hexadecimal (base-16). We focus on representations of these number systems together with arithmetical operations defined on them. The study of this paper ends with the conversions of number from one system into another with examples.
\end{abstract}

Key words: Arithmetic operations, binary system, decimal system, hexadecimal system, octal system,

\section{Introduction}

In a positional number system, there are only a few symbols called digits. A digit is a numeral symbol, used in combinations, to represent numbers in positional number systems. These symbols represent different values depending upon the position they occupy in a number (Joshi, \& Bhatt, 2016). The value of each digit in such a number is determined by three considerations: the digit itself; the position of the digit in the number; and the base or radix of the number system. In general, the relationship between a digit, its position and the base of the number system is expressed by the following formula;

\section{DIGIT $\times$ BASE ${ }^{\text {POSITION }}$}

Any number in positional number system may have two parts: left of the decimal point (called integral part) and right of the decimal point (called the fractional part) (Bajracharya, \& Shreshtha, 2069; Uprety, \& Durga, 2076).

\section{Number Systems and their representation}

There are several positional number system,namely, binary (base-2), ternary (base-3), quaternary (base-4), quinary (base-5), senary (base-6), octal(base-8), decimal (base-10), duodecimal (base-12), hexadecimal (base-16), vigesimal (base-20), hexadecimal (base-60). Among these the Babylonian numeral system,that is, hexadecimal numeral system (base-60) is credited as the first positional number system (Ifrah, 2000).

Here we discuss the four common positional number systems. They are: decimal, binary, octal and hexadecimal. 


\subsection{Decimal Number System}

The word decimal is derived from the Latin root decen (ten). Decimal number system has 10 digits from 0 to 9 . Every number represents with $0,1,2,3,4,5,6,7,8,9$ in this number system. The base of decimal number system is 10 as it has ten digits. Hence the notation for decimal number system becomes

$$
\text { DIGIT } \times 10^{\text {POSITION }}
$$

In decimal number system, successive positions to the left of the decimal point represents units or ones, tens, hundreds, thousands and so on and each position represents a specific power of the base, which is 10 . To represent a decimal fraction, successive positions to the right of the decimal point represent tenth, hundredth, thousandth and so on. The decimal number $(123.056)_{10}$ can be written as

$$
1 \times 10^{2}+2 \times 10^{1}+3 \times 10^{0}+0 \times 10^{-1}+5 \times 10^{-2}+6 \times 10^{-3}
$$

\subsection{Binary number system}

The word binary is derived by Latin root bini (two each). Binary Number System has only two digits 0 and 1 . Every number represents with 0 and 1 in this number system. The base of binary number system is 2 as it has two digits. Hence the notation for binary number system becomes

\section{DIGIT $\times 2^{\text {POSITION }}$}

In binary number system, successive positions to the left of the binary point represent units or ones, twos, fours and so on and each position represents a specific power of the base, which is 2 . To represent a binary fraction successive positions to the right of the binary point represents halves, fourths, eighths and so on. The binary number $110.11_{2}$ can be written as

\subsection{Octal number system}

$$
1 \times 2^{2}+1 \times 2^{1}+0 \times 2^{0}+1 \times 2^{-1}+1 \times 2^{-2}
$$

The word octal is derived from the Latin word octo(eight). Octal number system has eight digits from 0 to 7 . Every number represents with $0,1,2,3,4,5,6,7$ in this number system. The base of Octal number system is 8 as it has eight digits. Hence the notation for the octal number system becomes

$$
\text { DIGIT } \times 8^{\text {POSITION }}
$$

In octal number system, successive positions to the left of the octal points represents units, eighths, sixtyfours and so on and each position represents a specific power of the base which is 8 . To represent an octal fractions, successive positions to the right of the octal point represents eighths, sixty fourths as so on. The octal number $3733.45_{8}$ can be written as

$$
3 \times 8^{3}+7 \times 8^{2}+3 \times 8^{1}+3 \times 8^{0}+4 \times 8^{-1}+5 \times 8^{-2}
$$

\subsection{Hexadecimal number system}

The word hexadecimal is derived from Greek root hex(six) and the Latin root decen(ten). Hexadecimal number system has 16 alphanumeric values from 0 to 9 and A to F. Every number represents with $0,1,2,3,4,5,6,7,8,9, \mathrm{~A}, \mathrm{~B}, \mathrm{C}, \mathrm{D}, \mathrm{E}, \mathrm{F}$ in this number system. The base of hexadecimal number system is 16 as it has sixteen alphanumeric values. Here A is $10, \mathrm{~B}$ is $11, \mathrm{C}$ is $12, \mathrm{D}$ is 13 , E is 14 and $\mathrm{F}$ is 15 . Hence the notation for hexadecimal number system becomes

$$
\text { DIGIT } \times 16^{\text {POSITION }}
$$

In Hexadecimal number system, successive points to the left of the hexadecimal point represents units, sixteen and so on and each position represents a specific power of the base which is 16 . To represent a hexadecimal fraction, successive positions to the right of the hexadecimal point represents sixteenth, 
two hundred fifty sixths and so on. The hexadecimal number $2 \mathrm{~A} 3_{16}$ can be written as

$$
2 \times 16^{2}+\mathrm{A} \times 16^{1}+3 \times 16^{0}
$$

\section{Operations on different number system}

Arithmetic operation on decimal numbers are very popular and much easier. Here, arithmetic operations on binary, octal and hexadecimal numbers are explained.

\subsection{Addition:}

In Binary system, there are only two digits 0 and 1 . So, sum of two or more binary numbers cannot be 2 or more. If the sum is 2 , divide it by 2 then 1 is the quotient and the remainder is zero. So, in place of 2, write down 0 and 1 is the carry over. Thus, we have the following four rules.

$$
0+0=0,0+1=1,1+0=1 \text { and } 1+1=10 \text {. }
$$

In the case of Octal system, there are eight digits ranging from 0 to 7 . So, sum of two or more octal numbers cannot exceed 7 . If the sum is 8 , divide it by 8 , the quotient is 1 and the remainder is 0 . So, we write down 0 and 1 is the carry over. Thus follow the following rules.

$$
0+7=7,2+6=10,3+7=12 \text {, etc. }
$$

In the case of hexadecimal system, there are sixteen digits ranging from 0 to F. So, sum of two hexadecimal cannot exceed $15(=\mathrm{F})$. If the sum is 16 , divide it by 16 , the quotient is 1 and the remainder is 0 . So, we write down 0 and 1 is the carry over. Thus, the following rules are followed.

$$
0+9=9,1+9=\mathrm{A}, 8+8=10 \text { etc. }
$$

\subsection{Subtraction}

In binary system, the following four rules are obeyed for binary subtraction.

$$
0-0=0,1-0=1,1-1=0 \text { and } 0-1=0
$$

Borrow 1 is required from just higher position to subtract 1 from 0 . So, the result became 0 .In octal system, subtraction of octal number can be performed by the following rules of subtraction of decimal numbers but the digit borrow from the just higher position is counted as 8 . Thus, follow the following rules,

$$
4-0=4,6-5=1,7-7=0 \text { and } 2-4=6
$$

Borrow 1 is required from just higher position to subtract 4 from 2. So, the result became $8+2-4=6$.

In hexadecimal system, subtraction of hexadecimal numbers can be performed as decimal subtractions but the digit borrow from the just higher position is counted as 16. Thus, follow the following rules.

$$
6-3=3, C-7=5, D-B=2 \text { and } C-D=F
$$

Borrow 1 which is counted as 16 is required from just higher position to subtract $\mathrm{D}$ from $\mathrm{C}$. So result became $16+12-13=\mathrm{F}$.

\subsection{Multiplication}

In binary system, the following four rules are obeyed for binary multiplication

$$
0 \times 0=0,0 \times 1=0,1 \times 0=0,1 \times 1=1
$$

In octal system, if the product of octal numbers is less than 8 , then it is taken as a result, otherwise it is divided by 8 and the remainder is taken as least significant bit. The quotient is taken as carry in the next significant digit. Thus the following rules

$$
0 \times 0=0,2 \times 3=6,5 \times 2=12,15 \times 3=47
$$


In hexadecimal system, if the product of hexadecimal numbers is less than 16 , then it is taken as a result, otherwise it is divided by 16 and the remainder is taken as least significant bit. The quotient is taken as carry in the next significant digit. Thus, follow the following rules

\subsection{Division}

$$
0 \times 0=0,2 \times 4=8,3 \times 4=\mathrm{C}, 25 \times \mathrm{A}=172
$$

Division of binary, octal and hexadecimal numbers can be performed by the following rules of division of decimal numbers. Note that the maximum allowed digit in binary, octal and hexadecimal are 1, 7 and $\mathrm{F}(=15)$ respectively.

\section{Conversions of numbers from one system into another}

\subsection{Conversion of Decimal system to other systems:}

\subsubsection{Decimal to Binary}

To convert integral part of the decimal number to the binary number, we use the repeated process of division by 2. For this, we divide the given decimal number by 2 and we write down the remainder on the side. The process is continued by dividing the quotient by 2 and dropping the pervious remainders until the quotient is zero. Then the remainders listed from bottom to top will give the required corresponding binary number.

To convert the fractional part of the decimal numerals to binary form, the fractional part is multiplied by 2 and the integer obtained will be the first digit of the fractional part of the binary form. The remaining fractional part is again multiplied by 2 and the integer obtained will be the second digit of fractional part of binary form. This process is continued until the fractional part of the numeral is null. Example 1: Convert (75.6875) $)_{10}$ to Binary.

For integral part

Here, base is 2

For Fractional Part

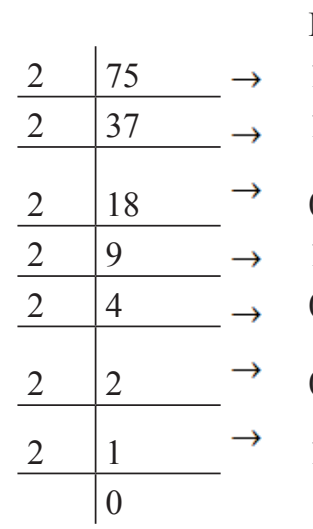

Remainder

\begin{tabular}{|c|c|c|}
\hline 2 & 75 & $\rightarrow$ \\
\hline 2 & 37 & $\rightarrow$ \\
\hline 2 & 18 & $\rightarrow$ \\
\hline 2 & 9 & $\rightarrow$ \\
\hline 2 & 4 & $\rightarrow$ \\
\hline 2 & 2 & $\rightarrow$ \\
\hline \multirow[t]{2}{*}{2} & 1 & $\rightarrow$ \\
\hline & 0 & \\
\hline
\end{tabular}

$\therefore(75.6875)_{10}=(1001011.1011)_{2}$

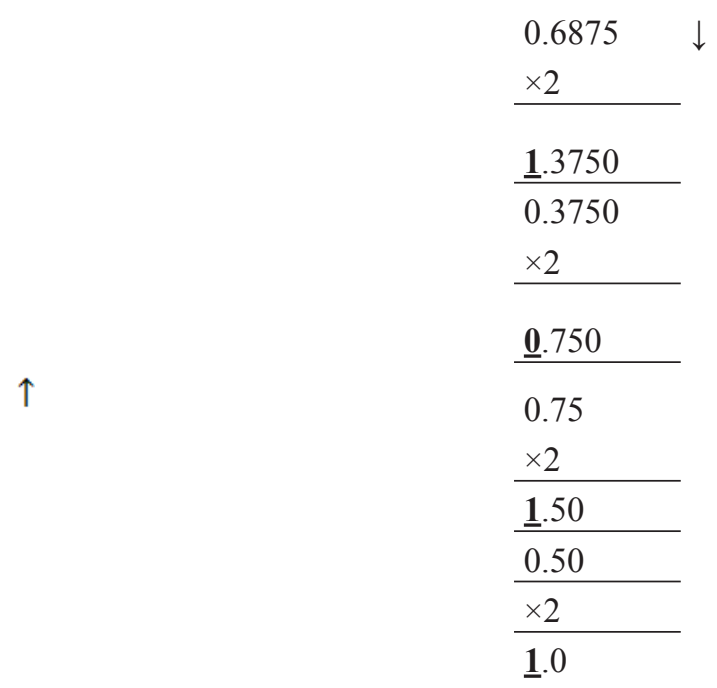




\subsubsection{Decimal to Octal}

To convert decimal number to an octal number, replace 2 by 8 in (3.1.1).

Example 2: Convert (86.25) $)_{10}$ to Octal.

For integral part

Here, base is 8

For Fractional Part

\begin{tabular}{|c|c|c|c|}
\hline 8 & 86 & $\rightarrow$ & Remainder \\
\hline 8 & 10 & $\rightarrow$ & 6 \\
\hline 8 & 1 & $\rightarrow$ & 2 \\
\hline & $\begin{array}{c}0 \\
86.2\end{array}$ & $\rightarrow$ & $\begin{array}{c}1 \\
126.2)\end{array}$ \\
\hline
\end{tabular}

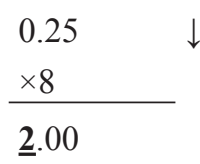

\subsubsection{Decimal to hexadecimal}

To convert decimal number to hexadecimal number, replace 2 by 16 in (3.1.1).

Example 3: Convert (7551.625) ${ }_{10}$ to Hexadecimal

For integral part

Here, base is 16

For Fractional Part

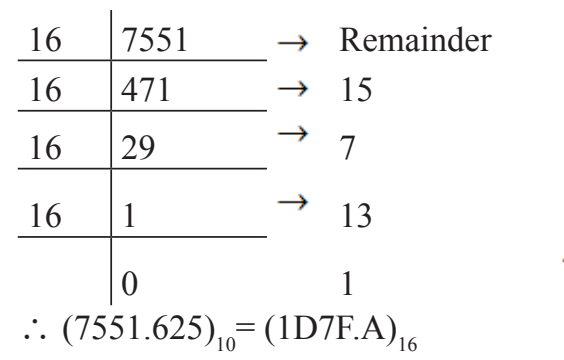

$0.625 \downarrow \downarrow$

$\times 16$

$\underline{\mathbf{A}}$

\subsection{Conversion of other systems to Decimal System}

\subsubsection{Binary to Decimal}

To convert integral part of binary number to the decimal number, each digit of the binary number is multiplied by a power of two, the power depending upon the position of the digit. The products are then added together to get the number in the decimal system. Again, to convert fractional part of the binary numerals, we change it to decimal form by multiplying the first digit of the fractional part by $1 / 2$, next digit by $1 / 2^{2}$, then $1 / 2^{3}$ and so on and then adding up the results will give the fractional part of the number in decimal form.

Example 4: Convert (10101.11001) $)_{2}$ into decimal form.

$$
\begin{aligned}
& 10101.11001=1 \times 2^{4}+0 \times 2^{3}+1 \times 2^{2}+0 \times 2^{1}+1 \times 2^{0}+1 \times 1 / 2^{1}+1 \times 1 / 2^{2}+0 \\
& \times 1 / 2^{3}+\times 1 / 2^{4} \\
& =16+0+4+0+1+0.5+0.25+0+0.00625 \\
& =21.8125
\end{aligned}
$$


$\therefore(10101.11001)_{2}=(21.8125)_{10}$

\subsubsection{Octal to decimal}

To convert an octal number to a decimal number, replace 2 by 8 in (3.2.1)

Example 5: Convert 112.2 into decimal form.

$112.2=1 \times 8^{2}+1 \times 8^{1}+2 \times 8^{0}+2 \times 1 / 8$

$=74.25$

$\therefore(112.2)_{8}=(74.25)_{10}$

\subsubsection{Hexadecimal to decimal}

To convert a hexadecimal to a decimal number, replace 2 by 16 in (3.2.1).

Example 6: Convert (2D4AC.2) ${ }_{16}$ into decimal form.

$2 D 4 A C .2=2 \times 16^{4}+13 \times 16^{3}+4 \times 16^{2}+10 \times 16^{1}+12 \times 16^{0}+2 \times 1 / 16$

$=185516.125$

$\therefore(2 D 4 A C .2)_{16}=(185516.125)_{10}$

\subsection{Conversion of Binary to Octal and Hexadecimal}

\subsubsection{Binary to Octal}

A binary number can be converted into its octal equivalent by using two methods (Mishra, \& Sigdel, 2068) :

Method I: For converting a binary number to its octal equivalents, we need to convert binary number to decimal form as in (3.2.1) and then convert decimal to octal form as in (3.1.2).

Method II: We can also convert the binary to octal number by grouping three binary digits to produce a signal octal number. Grouping can be done from right to left for integral part but for fractional part grouping is done from left to right. The following table will be helpful.

\begin{tabular}{|l|l|l|l|l|l|l|l|l|}
\hline 3-bit binary number & 000 & 001 & 010 & 011 & 100 & 101 & 110 & 111 \\
\hline Octal number & 0 & 1 & 2 & 3 & 4 & 5 & 6 & 7 \\
\hline
\end{tabular}

Example 7: Convert (110011101.11) into octal number.

Method I: First of all, the given number can be converted into decimal number as in example (4) and then decimal number into octal number as in example (2).

\subsubsection{Binary to Hexadecimal}

Method II: 110011101.11$)_{2}=110011101.110$ (grouping of 3$)=(635.6)_{8}$

A binary number can be converted into its hexadecimal equivalent by using two methods:

Method I: For converting a binary number to its hexadecimal equivalents, we need to convert decimal to hexadecimal form as in (3.2.1) and then convert decimal to hexadecimal form as in (3.1.3).

Method II: We can also convert the binary to hexadecimal by grouping four binary digits to produce a single hexadecimal number. Grouping is done from right to left for integral part whereas from left to right for the fractional part. The following table will be helpful. 


\begin{tabular}{|l|l|l|l|l|l|l|l|l|}
\hline 4-bit binary number & 0000 & 0001 & 0010 & 0011 & 0100 & 0101 & 0110 & 0111 \\
\hline Hexadecimal Number & 0 & 1 & 2 & 3 & 4 & 5 & 6 & 7 \\
\hline & 1000 & 1001 & 1010 & 1011 & 1100 & 1101 & 1110 & 1111 \\
\cline { 2 - 9 } & 8 & 9 & A & B & C & D & E & F \\
\hline
\end{tabular}

Example 8: Convert (11101100111.101) 2 to hexadecimal form

Method I: First of all, the given number can be converted into decimal number as in example (4) and then decimal number into hexadecimal number as in example (3).

Method II:

$(11101100111.101)_{2}=011101100111.1010($ grouping of 4$)$ $=(767 . A)_{16}$

\subsection{Conversion of Octal to Binary and Hexadecimal}

\subsubsection{Octal to Binary}

An Octal number can be converted into its binary equivalent by using two methods:

Method I: For converting an octal number into its binary form, we need to first convert octal number to decimal form as in (3.2.2) and then convert decimal to binary form as in (3.1.1).

Method II: We can also convert the octal to binary by converting octal digit into 3-bit binary and arrange in order from right to left for integral part and from left to right for fractional part.

Example 9: Convert $156_{8}$ to binary.

Method I: First of all, the given can be converted into decimal number as in example (5) and then decimal number into binary number as in example (1).

Method II:

3-bit binary numbers for each digit of $156_{8}$ are

$1_{8}=001_{2}, 5_{8}=101_{2}, \quad 6_{8}=110_{2}$

$\therefore 156_{8}=001101110=001101110=1101110_{2}$

\subsubsection{Octal to Hexadecimal}

An octal number can be converted into its hexadecimal equivalent by using the following two methods:

Method I: For converting an octal number to its hexadecimal form, we need first convert octal number to decimal form as in (3.2.2) and then convert decimal to hexadecimal form as in (3.1.3).

Method II: We can convert the octal to hexadecimal by converting octal digit into 3-bit binary and then regrouping them as binary quadruplets (4-bit binary number) and finally convert each group into its equivalent hexadecimal digit.

Example (10) Convert (1057) ${ }_{8}$ into hexadecimal number.

Method I: The given number can be converted into decimal number as in example (5) and then decimal number into hexadecimal number as in example (3).

Method II:

$(1057)_{8}=001000101111$ (grouping of 3)

NUTA JOURNAL, 7 (1 \& 2), 2020 : ISSN: 2616 - 017x 
$=001000101111$ (grouping of 4 )

$=22 F_{16}$

\subsection{Conversion of Hexadecimal to binary and octal}

\subsubsection{Hexadecimal to Binary}

A hexadecimal number can be converted into its binary equivalent by using two methods:

Method I: For converting a hexadecimal number to its binary form, we need first convert hexadecimal number to decimal form as in (3.2.3) and then convert decimal to binary form as in (3.1.1).

Method II: We can convert the hexadecimal to binary by converting hexadecimal digit into 4-bit binary and arrange in order from right to left for integral part and from left to right for fractional part.

Example 11: Convert AFB2 16 to binary form

Method I: First of all, the given number can be converted into decimal number as in example (6) and then decimal number in to binary number as in example (1).

Method II:

4-bit binary numbers for each digit of $\mathrm{AFB} 2_{16}$ are

$A=1010_{2}, F=1111_{2}, B=1011_{2}, 2=0010_{2}$

$\therefore A F B 2_{16}=1010111110110010=1010111110110010_{2}$

\subsubsection{Hexadecimal to Octal:}

A hexadecimal number can be converted into its octal equivalent by using the following two methods:

Method I: For converting a hexadecimal number to its octal form, we need first convert hexadecimal number to decimal form as in (3.2.3) and then convert decimal number to octal form as in (3.1.2).

Method II: we can convert the hexadecimal to octal by converting hexadecimal digit into 4-bit binary and then regrouping them as binary triplets ( 3 bit binary number) and finally convert each group into its equivalent octal digit.

Example 12: Convert $(\mathrm{E} 3 \mathrm{~F})_{16}$ to an octal number.

Method I: First of all the given number can be converted into decimal number as in example (6) and the decimal number into octal number as in example (2).

Method II:

$(E 3 F)_{16}=111000111111$ (grouping of 4)

$=111000111111$ (grouping of 3 )

$$
=(7077)_{8}
$$

\section{Conclusion}

This paper is devoted to giving the representation of decimal, binary, octal and hexadecimal system, the operations on these number system and methods for converting one system to another system with different examples. It is concluded that the representation of positional number systems shows that there is a fixed place or position for each and every discrete element. These elements always represent a discrete value. The value of a certain element depends on the specific position of that element, base of 
the number systems and also on the element itself. With positional notation, arithmetical computations are greatly simpler and this explains the rapid spread of the notation. The conversion of one system to another number system with higher base can store much higher values into far fewer characters

\section{References:}

Bajracharya, D.R., Shrestha, R.M., et al.(2069). Basic Mathmatics, Grade XII, Sukunda Pustak Bhawan, Kathmandu.

Ifrah,G., (2000). The Universal History of numbers: From prehistory to the invension of the computers. John Wiley \& Sons, Inc.

Joshi, C., Bhatta C.R., et al (2016). Essentials of Mathematics, Grade XII, $2^{\text {nd }}$ edition, Ayam Publication and Distributors Pvt. Ltd., Kathmandu.

Mishra, S.K., Sigdel, D.R. et al (2068). Conceptual Mathematics, Grade XII, $6^{\text {th }}$ ed., Divya Deurali Prakashan Pvt. Ltd, Kathmandu.

Uprety, K.N, K.C, Durga J. et al. (2076). Foundations of Mathematics, Grade XII, Asmita Books Publishers and Distributors Pvt. Ltd., Kathmandu.

\section{Websites:}

https://danielmiessler.com/study

https://www.tutorialpoint.com/arithmeticofnumbersystems

https://www.sciencedirect.com/book

https://www.elseveir.com/klose

https://www.weddocos.cs.ualberta.ca

https://www.researchgate.net

https://www.cs.princeton.edu

https://www.includehelp.com/computernumbersystem 